\title{
On the Probability, Rate, and Duration of Phase Outages in Nakagami Fading Channels
}

\author{
José Cândido Silveira Santos Filho and Michel Daoud Yacoub
}

\begin{abstract}
A plausible phase-envelope model has been recently proposed for Nakagami fading. In contrast to the usual assumption that the phase is uniform, the model leads to phase statistics that change according to the Nakagami fading parameter. Based on this model, we derive exact closed-form expressions for the probability, average rate, and average duration of phase outages in Nakagami fading channels. The new expressions reveal that the fading parameter has a deep impact on the Nakagami phase statistics.
\end{abstract}

Keywords - Nakagami fading, outage duration, outage probability, outage rate, phase.

\section{INTRODUCTION}

W HEN proposed, the Nakagami fading model specified the distribution of the channel envelope but not of the channel phase [1]. Because of this, and knowing that the phase distribution of Rayleigh fading - a special case of Nakagami fading - is uniform, researchers have arbitrarily assigned a uniform distribution to the phase of Nakagami channels. Recently, a plausible phase-envelope model was proposed for Nakagami fading that accounts for the effects of the fading parameter on both the phase and envelope of the channel [2]. In that model, the envelope follows the well-known Nakagami distribution, but the phase is no longer uniform, except for the special Rayleigh case. The primary motivation for the use of the Nakagami fading model proposed in [2] is that its phase distribution (i) coincides with those of Rice and Hoyt for the limiting cases in which their envelope distributions also coincide (ii) and bears similar shapes to those of Rice and Hoyt for the remaining cases, in which their envelope distributions approach each other.

The probability, rate, and duration of phase outages are of interest in some wireless communication applications, including carrier recovery schemes in coherent receivers [3] and frequency-modulation receivers using a limiter discriminator [4]. The phase statistics have been studied in [5] for Rice fading, in [6] for Hoyt fading, and in [2] and [7] for Nakagami fading. More specifically, [2] derived the probability density function (PDF) of the Nakagami phase, and [7] derived its average crossing rate (ACR). In the present work, we complete the studies in [2] and [7] by deriving exact closedform expressions for the outage probability (OP), average outage rate $(\mathrm{AOR})^{1}$, and average outage duration $(\mathrm{AOD})$ of the Nakagami phase. The new expressions reveal that the fading parameter has a deep impact on the Nakagami phase statistics.

The authors are with the Department of Communications, School of Electrical and Computer Engineering, University of Campinas, PO Box 6101, 13083-852 Campinas, SP, Brazil, tel: +55 (19) 3521 5106, fax: +55 (19) 3289 1395, e-mail:[candido,michel]@wisstek.org.

${ }^{1}$ For phase processes, there is a crucial distinction between ACR and AOR, as detailed in Section III.

\section{Probability, Rate, And Duration of Phase OUTAGES IN NAKAGAMI FADING CHANNELS}

The OP of a random signal is defined as the probability that the signal is less than or equal to a given level ${ }^{2}$; the AOR is defined as the mean rate at which the signal falls below that level; and the AOD is defined as the mean time the signal remains under the level after falling bellow it. Next, we show that the OP $F_{\Theta}(\theta)$, the AOR $N_{\Theta}(\theta)$, and the AOD $T_{\Theta}(\theta)$ of the Nakagami phase $\Theta$ are given by

$$
\begin{aligned}
& F_{\Theta}(\theta)=\frac{1}{8} \sum_{i=1}^{8}\left[\left(\lfloor i\rfloor_{o d d}+\frac{(-1)^{i} \Gamma\left(\frac{m+1}{2}\right) \mathrm{B}_{\cos ^{2}(2 \theta)}\left(\frac{1}{2}, \frac{m}{2}\right)}{\sqrt{\pi} \Gamma\left(\frac{m}{2}\right)}\right)\right. \\
& \left.\times \Pi\left(\frac{4 \theta}{\pi}+\frac{9}{2}-i\right)\right] \\
& N_{\Theta}(\theta)=\frac{\sqrt{\pi} f_{D} b(m) \Gamma\left(m-\frac{1}{2}\right)|\sin (2 \theta)|^{m-1}}{2^{m+\frac{1}{2}} \Gamma\left(\frac{m}{2}\right)^{2}} \\
& T_{\Theta}(\theta)=\frac{|\sin (2 \theta)|^{1-m}}{4 \sqrt{2 \pi} f_{D} b(m) \Gamma\left(m-\frac{1}{2}\right)} \\
& \times \sum_{i=1}^{8}\left[\left(2^{m} \Gamma\left(\frac{m}{2}\right)^{2}\lfloor i\rfloor_{o d d}+2(-1)^{i} \Gamma(m) \mathrm{B}_{\cos ^{2}(2 \theta)}\left(\frac{1}{2}, \frac{m}{2}\right)\right)\right. \\
& \left.\times \Pi\left(\frac{4 \theta}{\pi}+\frac{9}{2}-i\right)\right] \\
& -\pi \leq \theta<\pi \\
& \Pi(x) \triangleq \begin{cases}1 & -\frac{1}{2} \leq x<\frac{1}{2} \\
0 & \text { otherwise }\end{cases} \\
& b(m) \triangleq\left\{\begin{array}{ll}
\infty & \frac{1}{2} \leq m<1 \\
2 & m=1 \\
1 & m>1
\end{array},\right.
\end{aligned}
$$

where $m$ is the Nakagami fading parameter, $f_{D}$ is the maximum Doppler shift in $\mathrm{Hz}, \Gamma(\cdot)$ is the gamma function, $\mathrm{B}_{(\cdot)}(\cdot, \cdot)$ is the incomplete beta function, and $\lfloor x\rfloor_{o d d}$ is the greatest odd integer less than or equal to $x$.

\section{DERIVATION}

Our first task is to evaluate the outage probability, defined as

$$
F_{\Theta}(\theta) \triangleq \int_{-\pi}^{\theta} f_{\Theta}(u) d u,-\pi \leq \theta<\pi
$$

\footnotetext{
${ }^{2}$ The outage probability is equivalent to the cumulative distribution function.
} 
where $f_{\Theta}(\theta)$ is the Nakagami phase PDF [2]

$$
f_{\Theta}(\theta)=\frac{\Gamma(m)|\sin (2 \theta)|^{m-1}}{2^{m} \Gamma\left(\frac{m}{2}\right)^{2}},-\pi \leq \theta<\pi .
$$

In order to solve (2), we shall exploit the periodicity and symmetry of $f_{\Theta}(\theta)$ as given in (3). Owing to the term $|\sin (2 \theta)|$, the Nakagami phase PDF is periodic with period $\pi / 2$, and has a repetitive pattern within the period, since $|\sin (2 \theta)|=|\sin (2(\pi / 2-\theta))|$. Thus, the phase PDF is fully characterized by its behavior within a half period $\pi / 4$, e.g., $0 \leq \theta \leq \frac{\pi}{4}$. As a consequence, any probability mass of the Nakagami phase can be expressed in terms of its probability mass $p(\theta)$ over $0 \leq \theta \leq \frac{\pi}{4}$, defined as

$$
p(\theta) \triangleq \operatorname{Pr}[0<\Theta \leq \theta]=\int_{0}^{\theta} f_{\Theta}(u) d u, 0 \leq \theta \leq \frac{\pi}{4},
$$

where $\operatorname{Pr}[\cdot]$ denotes probability.

The integral in (4) can be evaluated in an exact closed form, yielding

$$
p(\theta)=\frac{1}{8}\left[1-\frac{\Gamma\left(\frac{m+1}{2}\right) \mathrm{B}_{\cos ^{2}(2 \theta)}\left(\frac{1}{2}, \frac{m}{2}\right)}{\sqrt{\pi} \Gamma\left(\frac{m}{2}\right)}\right] .
$$

Then, using the above-mentioned periodicity and symmetry of $f_{\Theta}(\theta), F_{\Theta}(\theta)$ can be evaluated by piecewise integration and written in terms of $p(\theta)$ as

$$
F_{\Theta}(\theta)= \begin{cases}p(\theta+\pi) & -\pi \leq \theta<\frac{3 \pi}{4} \\ \frac{1}{4}-p\left(-\theta-\frac{\pi}{2}\right) & -\frac{3 \pi}{4} \leq \theta<-\frac{\pi}{2} \\ \frac{1}{4}+p\left(\theta+\frac{\pi}{2}\right) & -\frac{\pi}{2} \leq \theta<-\frac{\pi}{4} \\ \frac{1}{2}-p(-\theta) & -\frac{\pi}{4} \leq \theta<0 \\ \frac{1}{2}+p(\theta) & 0 \leq \theta<\frac{\pi}{4} \\ \frac{3}{4}-p\left(\frac{\pi}{2}-\theta\right) & \frac{\pi}{4} \leq \theta<\frac{\pi}{2} \\ \frac{3}{4}+p\left(\theta-\frac{\pi}{2}\right) & \frac{\pi}{2} \leq \theta<\frac{3 \pi}{4} \\ 1-p(\pi-\theta) & \frac{3 \pi}{4} \leq \theta<\pi\end{cases}
$$

Although $p(\theta)$ was originally defined in (4) within the range $0 \leq \theta \leq \pi / 4$, it may be now useful to extend the domain of the resulting expression (5) to $-\pi \leq \theta<\pi$; by doing so, simplifications in (6) are attained, as follows. Note in (5) that $p(\theta)$ depends on $\cos ^{2}(2 \theta)$, which-similarly to the phase PDF-is periodic with period $\pi / 2$ and symmetric within the period. In particular, $p(\theta)=p(-\theta)=p(\theta \pm \pi / 2)=p(\theta \pm \pi)$. Using this and relaxing the domain of $p(\theta)$ in (5) to $-\pi \leq$ $\theta<\pi$, (6) can be simplified to

$$
F_{\Theta}(\theta)=\left\{\begin{array}{ll}
p(\theta) & -\pi \leq \theta<\frac{3 \pi}{4} \\
\frac{1}{4}-p(\theta) & -\frac{3 \pi}{4} \leq \theta<-\frac{\pi}{2} \\
\frac{1}{4}+p(\theta) & -\frac{\pi}{2} \leq \theta<-\frac{\pi}{4} \\
\frac{1}{2}-p(\theta) & -\frac{\pi}{4} \leq \theta<0 \\
\frac{1}{2}+p(\theta) & 0 \leq \theta<\frac{\pi}{4} \\
\frac{3}{4}-p(\theta) & \frac{\pi}{4} \leq \theta<\frac{\pi}{2} \\
\frac{3}{4}+p(\theta) & \frac{\pi}{2} \leq \theta<\frac{3 \pi}{4} \\
1-p(\theta) & \frac{3 \pi}{4} \leq \theta<\pi
\end{array} .\right.
$$

It may be useful to rewrite the piecewise solution in (7) as a sum of shifted window functions that can be directly implemented in most computing softwares. Performing thisafter some algebraic manipulations - the OP of the Nakagami phase is finally obtained as in (1a).
Our second task is to evaluate the average outage rate. At this point, we distinguish the average outage rate $N_{\Theta}(\theta)$ - the mean rate at which the signal falls bellow a given level-from the (more usual) average crossing rate $n_{\Theta}(\theta)$ - the mean rate at which the signal actually down-crosses that level. For timecontinuous signals, e.g. the channel envelope, the distinction is pointless; the only way for a time-continuous signal to fall bellow a given level is by down-crossing it. However, for timediscontinuous signals, e.g. the channel phase, the distinction is pertinent; such a signal may fall bellow a given level both by down-crossing that level or by jumping over it through signal discontinuities. In particular, the phase signal is (by definition) limited to $-\pi \leq \theta<\pi$. Thus, phase crossings at $-\pi$ correspond to abrupt phase shifts of $2 \pi$. And the point here is that, given any phase level $\theta$, the phase signal may fall bellow that level both by down-crossing it-which occurs at the rate of $n_{\Theta}(\theta)$ - or by up-crossing $-\pi$ - which occurs at the rate ${ }^{3}$ of $n_{\Theta}(-\pi)$. That is,

$$
N_{\Theta}(\theta)=n_{\Theta}(\theta)+n_{\Theta}(-\pi),-\pi \leq \theta<\pi .
$$

The ACR of the Nakagami phase has been derived in [7] as

$$
n_{\Theta}(\theta)=\frac{\sqrt{\pi} f_{D} \Gamma\left(m-\frac{1}{2}\right)|\sin (2 \theta)|^{m-1}}{2^{m+\frac{1}{2}} \Gamma\left(\frac{m}{2}\right)^{2}},-\pi \leq \theta<\pi \text {. }
$$

In particular, $n_{\Theta}(-\pi) \rightarrow \infty$ for $1 / 2 \leq m<1, n_{\Theta}(-\pi)=$ $n_{\Theta}(\theta)$ for $m=1$, and $n_{\Theta}(-\pi)=0$ for $m>1$. Using this, (8) can be rewritten as

$$
N_{\Theta}(\theta)=b(m) n_{\Theta}(\theta),-\pi \leq \theta<\pi,
$$

where $b(\cdot)$ is defined as in (1f). Replacing (9) into (10), we then obtain the AOR of the Nakagami phase as in (1b).

Finally, we have to evaluate the average outage duration. This quantity is well known to be given in terms of the outage probability and the average outage rate as

$$
T_{\Theta}(\theta)=\frac{F_{\Theta}(\theta)}{N_{\Theta}(\theta)} .
$$

Replacing (1a) and (1b) into (11), the Nakagami phase AOD is attained as in (1c).

\section{NumericAl RESUlts}

We now plot some curves to illustrate the new expressions. Figs. 1, 2, and 3 show the OP, AOR, and AOD of the Nakagami phase, respectively, for different values of the fading parameter. In the special Rayleigh case $(m=1)$, for which the phase PDF is uniform, the OP is a straight line from $(-\pi, 0)$ to $(\pi, 1)$, the AOR is $f_{D} / \sqrt{2}$ for any phase level, and the AOD is a straight line from $(-\pi, 0)$ to $\left(\pi, \sqrt{2} / f_{D}\right)$. From (8), the Rayleigh phase AOR has indeed to be the double of the Rayleigh phase ACR, which is $f_{D} /(2 \sqrt{2})$ for any phase level [7]. On the other hand, for $1 / 2 \leq m<1$, the Nakagami phase OP oscillates around the straight Rayleigh phase OP. In this case, since the ACR goes to infinity at $-\pi$, then from (8) the AOR also goes to infinity at any phase

\footnotetext{
${ }^{3}$ Following the rationale in [7], it can be shown that the Nakagami phase has identical up- and down-crossing rates. Thus, $n_{\Theta}(-\pi)$ is also the up-crossing rate at $-\pi$.
} 


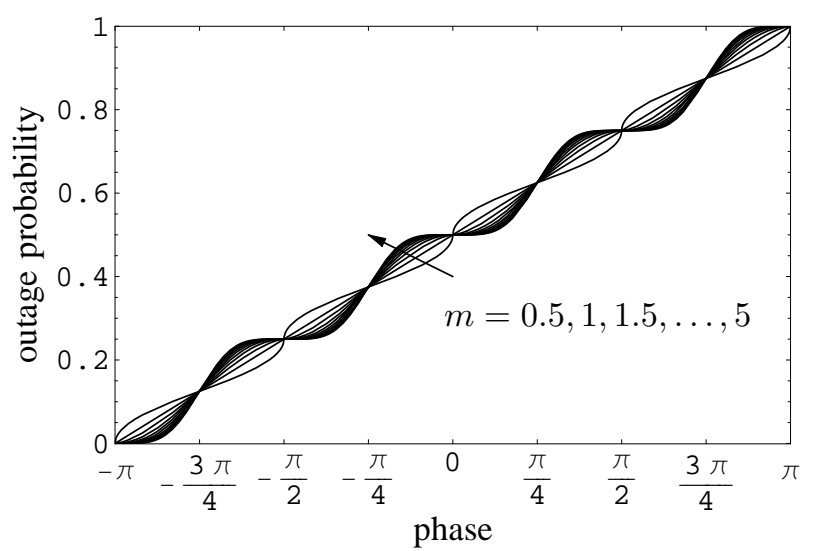

Fig. 1. Outage probability of the Nakagami phase.

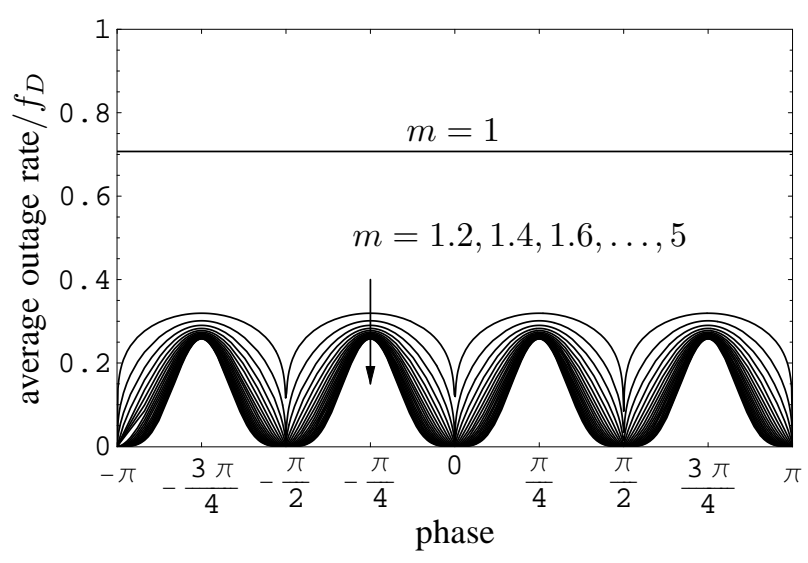

Fig. 2. Average outage rate of the Nakagami phase.

level. As a consequence, the AOD is nil. Finally, for $m>1$, the Nakagami phase OP again oscillates around the Rayleigh phase OP, although in the opposite direction when compared to the case $1 / 2 \leq m<1$. In this case, the AOR is identical to the ACR, because the latter is nil at $-\pi$. In addition, the AOR is also nil at $-\pi / 2,0$, and $\pi / 2$, as well as when it approaches $\pi$. Therefore, the AOD goes to infinity at these phase levels.

To gain insight into the fading phenomenon, we may infer from the curves the expected behavior of the Nakagami phasor signal. For $m=1$, the curves suggest that, on the average, the phasor signal rotates at constant speed, either clock- or counterclockwise, alternately. For $1 / 2 \leq m<1$, the phasor signal oscillates around $-\pi,-\pi / 2,0$, or $\pi / 2$ most frequently. In contrast, it rarely crosses these levels for $m>1$, remaining confined to one of the four quadrants.

\section{CONCLUSION}

We derived exact closed-form expressions for the probability, average rate, and average duration of phase outages in Nakagami fading channels. Contrarily to the usual assumption that the Nakagami phase is uniform, we showed that the fading parameter $m$ has a deep impact on the phase statistics. More specifically, we showed that, as $m$ increases, the phase outage

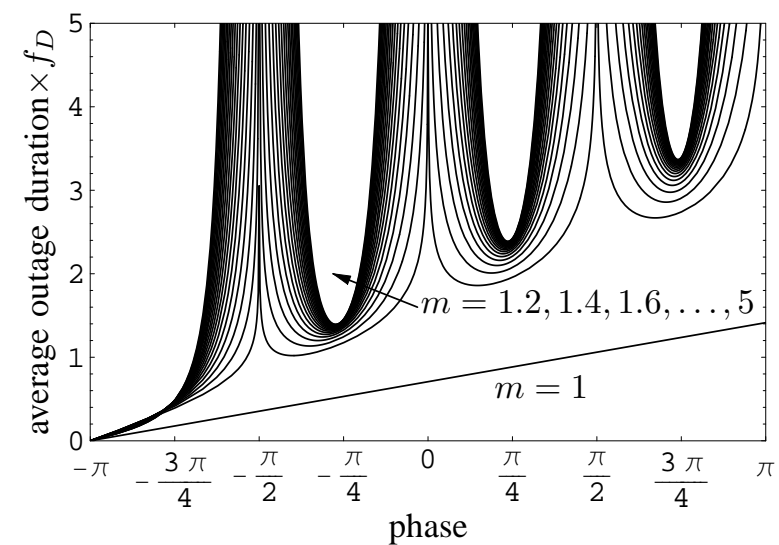

Fig. 3. Average outage duration of the Nakagami phase.

duration also increases, but the phase outage rate decreases. This is in contrast to the behavior of the channel envelope, for which both the outage rate and the outage duration are known to decrease (at low levels) as $m$ increases.

\section{REFERENCES}

[1] M. Nakagami, "The $m$-distribution - a general formula of intensity distribution of rapid fading," in Statistical Methods in Radio Wave Propagation, W. C. Hoffman, Ed. Oxford, England: Pergamon, 1960.

[2] M. D. Yacoub, G. Fraidenraich, and J. C. S. Santos Filho, "Nakagami$m$ phase-envelope joint distribution," Electron. Lett., vol. 41, no. 5, pp. 259-261, Mar. 2005.

[3] D. T. Hess, "Cycle slipping in a first order phase locked loop," IEEE Trans. Commun. Technol., vol. 16, pp. 255-260, Apr. 1968.

[4] R. G. Vaughan, "Signals in mobile communications: A review," IEEE Trans. Vehic. Technol., vol. 35, pp. 133-145, Nov. 1986.

[5] S. O. Rice, Noise in FM Receivers, ser. Time Series Analysis. New York: Wiley, 1963, ch. 25.

[6] N. Youssef, W. Elbahri, M. Pätzold, and S. Elasmi, "On the crossing statistics of phase processes and random FM noise in Nakagami-q mobile fading channels," IEEE Trans. Wireless Commun., vol. 4, no. 1, pp. 24-29, Jan. 2005.

[7] D. B. da Costa, M. D. Yacoub, J. C. S. Santos Filho, G. Fraidenraich, and J. R. Mendes, "Generalized Nakagami- $m$ phase crossing rate," IEEE Commun. Lett., vol. 10, no. 1, pp. 13-15, Jan. 2006. 